Title: Label-free Estimation of Therapeutic Efficacy on 3D Cancer Spheres Using Convolutional Neural Network Image Analysis

Authors and affiliations:

Zhixiong Zhang ${ }^{\dagger 1}$, Lili Chen ${ }^{\dagger 1}$, Yimin Wang ${ }^{1}$, Tiantian Zhang ${ }^{1}$, Yu-Chih Chen ${ }^{* 1,2}$, and Euisik Yoon ${ }^{* 1,3}$

${ }^{1}$ Department of Electrical Engineering and Computer Science, University of Michigan, 1301 Beal Avenue, Ann Arbor, MI 48109-2122;

${ }^{2}$ Forbes Institute for Cancer Discovery, University of Michigan, 2800 Plymouth Rd., Ann Arbor, MI 48109, USA;

${ }^{3}$ Department of Biomedical Engineering, University of Michigan, 2200 Bonisteel, Blvd. Ann Arbor, MI 48109-2099, USA

$\dagger$ These authors contributed equally to this work.

* Corresponding author

Yu-Chih Chen

1301 Beal Avenue, Ann Arbor, MI 48109-2122, USA

Tel: 734-272-7113; E-mail: yuchchen@umich.edu.

Euisik Yoon

1301 Beal Avenue, Ann Arbor, MI 48109-2122, USA

Tel: 734-615-4469; E-mail: esyoon@umich.edu. 


\section{Table of Content}

1. Supplementary Methods - Image Acquisition

2. Supplementary Methods - Neural Network Structure

3. Figure S1. Automatic sphere chamber cropping.

4. Figure S2. Neural network structure and trained filters for prediction.

5. Figure S3. Cancer spheres classification and drug inhibition score regression for Doxorubicin.

6. Figure S4. Cancer spheres classification and drug inhibition score regression for Oxaliplatin.

7. Figure S5. Cancer spheres classification and drug inhibition score regression for Oxaliplatin. 


\section{Supplementary Methods}

\section{Image Acquisition}

The microfluidic cancer sphere chips were imaged using an inverted microscope (Nikon). The brightfield and fluorescence images were taken with a $4 \mathrm{x}$ objective lens and a charge-coupled device (CCD) camera (Coolsnap HQ2, Photometrics). A FITC filter cube was used for the green fluorescence imaging of LIVE staining, and a TRITC filter cube was used for the red fluorescence imaging of DEAD staining. Brightfield microscopy was performed using an exposure time shorter than $10 \mathrm{~ms}$, and the fluorescence imaging was performed using an exposure time shorter than $300 \mathrm{~ms}$ to minimize phototoxic effects on cells. The device was scanned with a motorized stage (ProScan II, Prior Scientific). Auto focusing was used to ensure the image remained in focus throughout the whole imaging area.

\section{Neural Network Structure}

We designed a three-layer-set neural network structure for machine learning. Since the size of original cropped chamber image was too large ( 242 by 242 pixels) for convolutional neural network learning, the image was downsampled to 61 by 61 pixels. Within each convolutional layer set, there are 4 different layers: a convolutional layer, a batch normalization layer, a rectifier function layer, and a max pooling layer. The first convolutional layer has 64 filters with the size of 12 by 12 pixels. The second convolutional layer has 32 filters with the size of 6 by 6 pixels. The third convolutional layer has 16 filters with the size of 4 by 4 pixels. The max pooling layer in each convolutional layer set gets the maximum of a 2 by 2 rectangle from the image with the stride size of 2 . There is a dropout layer between the third convolutional layer set and the final fully connected layer. The dropout layer was used to avoid overfitting problem. For regression purpose, a regression layer was added after the fully connected layer. For classification purpose, a softmax layer followed by a classification layer was added after the fully connected layer. The network structure is illustrated as in Fig. S2. 


\section{Supplementary Figures}

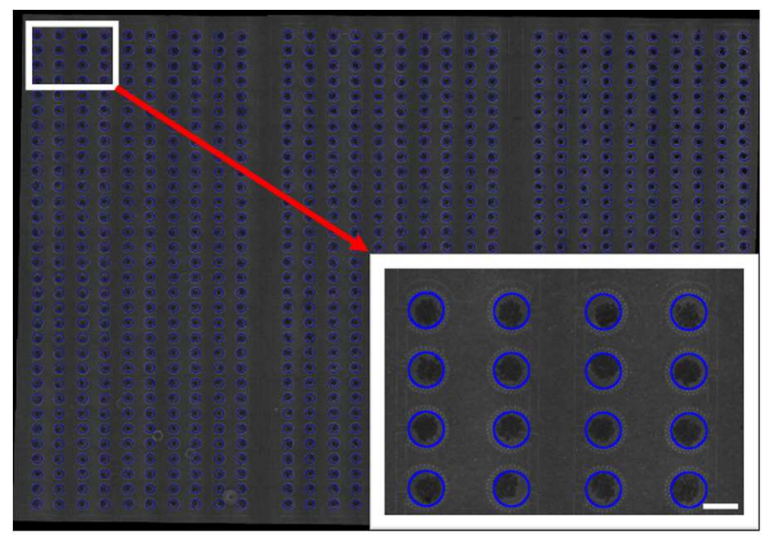

Figure S1. Automatic sphere chamber cropping. Sphere chamber cropping program can successfully crop chambers of the whole chip for data analysis and machine learning. The cropping circles are demonstrated as blue circles. (Scale bar: $400 \mu \mathrm{m}$ ) 

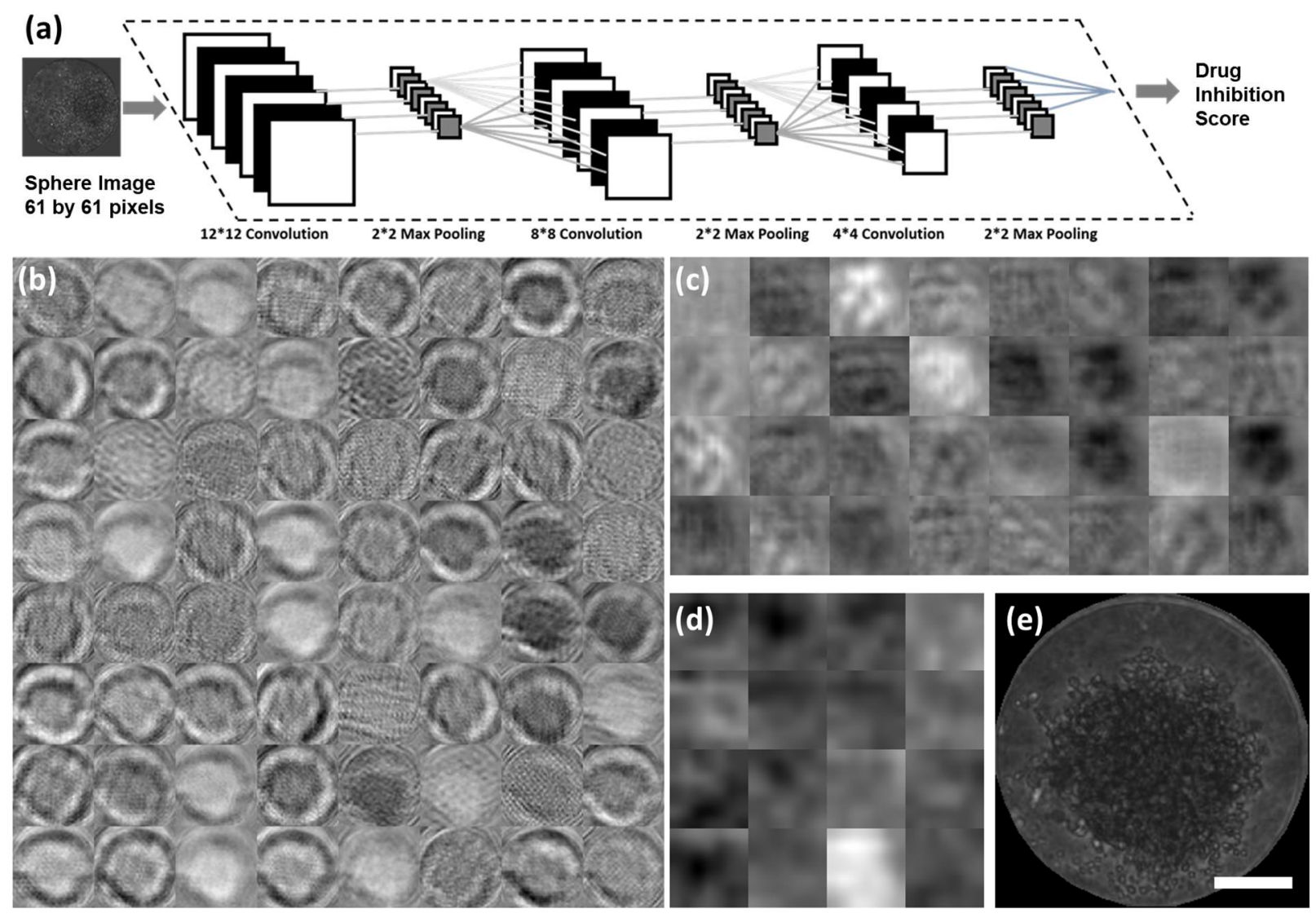

Figure S2. Neural network structure and trained filters for prediction (a) The 3-layer structure of neural network. (b-d) After processing a representative sphere image, the critical features are enhanced by filters of each convolutional layer in the neural network. The features are related to central darkness, sphere outline, and texture of cancer spheres. Each small square represents a filter. (b) The 64 filters in the first convolutional layer. (c) The 32 filters in the second convolutional layer. (d) The 16 filters in the final convolutional layer. (e) The input representative sphere image. (Scale bar: $100 \mu \mathrm{m}$ ) 

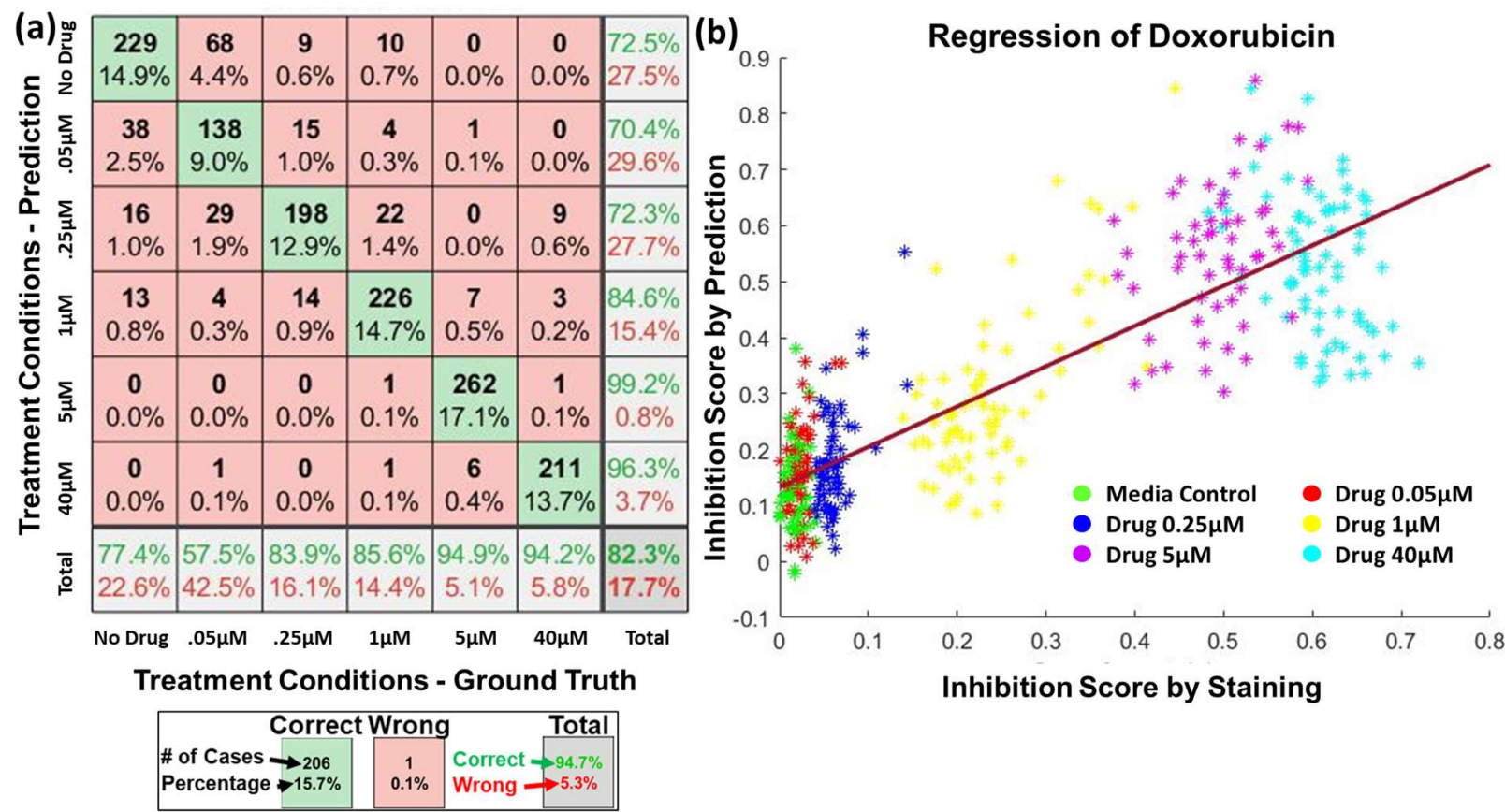

Figure S3. Cancer spheres classification and drug inhibition score regression for Doxorubicin. (a) Six-class classification accurately $(82.3 \%)$ predicts the drug treatment concentration based on its brightfield image. (b) Regression of sphere inhibition score using the trained CNN model. X-axis represents the ground truth of inhibition score measured by LIVE/DEAD staining, and Y-axis represents drug inhibition score predicted using bright-field image and trained model. Each dot indicates a sphere, and different colors mean different drug concentrations. The R-value of this linear regression is 0.84 , indicating a strong correlation between the ground truth and our prediction. 


\begin{tabular}{|c|c|c|c|c|c|c|c|}
\hline o & $\begin{array}{c}218 \\
14.2 \%\end{array}$ & $\begin{array}{c}70 \\
4.6 \%\end{array}$ & $\begin{array}{c}0 \\
0.0 \%\end{array}$ & $\begin{array}{c}\mathbf{0} \\
0.0 \%\end{array}$ & $\begin{array}{c}\mathbf{0} \\
0.0 \%\end{array}$ & $\begin{array}{c}\mathbf{0} \\
0.0 \%\end{array}$ & $\begin{array}{l}75.7 \% \\
24.3 \%\end{array}$ \\
\hline 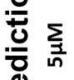 & $\begin{array}{c}86 \\
5.6 \%\end{array}$ & $\begin{array}{c}190 \\
12.4 \%\end{array}$ & $\begin{array}{c}0 \\
0.0 \%\end{array}$ & $\begin{array}{c}1 \\
0.1 \%\end{array}$ & $\begin{array}{c}0 \\
0.0 \%\end{array}$ & $\begin{array}{c}0 \\
0.0 \%\end{array}$ & $\begin{array}{l}68.6 \% \\
31.4 \%\end{array}$ \\
\hline 言 & $\begin{array}{c}0 \\
0.0 \%\end{array}$ & $\begin{array}{c}0 \\
0.0 \%\end{array}$ & $\begin{array}{c}\mathbf{2 1 4} \\
13.9 \%\end{array}$ & $\begin{array}{c}19 \\
1.2 \%\end{array}$ & $\begin{array}{c}4 \\
0.3 \%\end{array}$ & $\begin{array}{c}0 \\
0.0 \%\end{array}$ & $\begin{array}{c}90.3 \% \\
9.7 \%\end{array}$ \\
\hline 言 & $\begin{array}{c}0 \\
0.0 \%\end{array}$ & $\begin{array}{c}0 \\
0.0 \%\end{array}$ & $\begin{array}{c}11 \\
0.7 \%\end{array}$ & $\begin{array}{c}196 \\
12.8 \%\end{array}$ & $\begin{array}{c}12 \\
0.8 \%\end{array}$ & $\begin{array}{c}1 \\
0.1 \%\end{array}$ & $\begin{array}{l}89.1 \% \\
10.9 \%\end{array}$ \\
\hline 产 & $\begin{array}{c}\mathbf{0} \\
0.0 \%\end{array}$ & $\begin{array}{c}0 \\
0.0 \%\end{array}$ & $\begin{array}{c}11 \\
0.7 \%\end{array}$ & $\begin{array}{c}32 \\
2.1 \%\end{array}$ & $\begin{array}{c}\mathbf{2 0 4} \\
13.3 \%\end{array}$ & $\begin{array}{c}0 \\
0.0 \%\end{array}$ & $\begin{array}{l}82.6 \% \\
17.4 \%\end{array}$ \\
\hline 吾 & $\begin{array}{c}\mathbf{0} \\
0.0 \%\end{array}$ & $\begin{array}{c}0 \\
0.0 \%\end{array}$ & $\begin{array}{c}0 \\
0.0 \%\end{array}$ & $\begin{array}{c}0 \\
0.0 \%\end{array}$ & $\begin{array}{c}0 \\
0.0 \%\end{array}$ & $\begin{array}{c}267 \\
17.4 \%\end{array}$ & $\begin{array}{l}100 \% \\
0.0 \%\end{array}$ \\
\hline & $\begin{array}{l}71.7 \% \\
28.3 \%\end{array}$ & $\begin{array}{l}73.1 \% \\
26.9 \%\end{array}$ & $\begin{array}{c}90.7 \% \\
9.3 \%\end{array}$ & $\begin{array}{l}79.0 \% \\
21.0 \%\end{array}$ & $\begin{array}{c}92.7 \% \\
7.3 \%\end{array}$ & $\begin{array}{c}99.6 \% \\
0.4 \%\end{array}$ & $\begin{array}{l}83.9 \% \\
16.1 \%\end{array}$ \\
\hline & No Dr & & & & & & \\
\hline
\end{tabular}

\begin{tabular}{|c|c|c|}
\hline \multicolumn{3}{|c|}{ Correct Wrong } \\
\hline $\begin{array}{l}\text { \# of Cases } \rightarrow 206 \\
\text { Percentage } \rightarrow 15.7 \%\end{array}$ & $\begin{array}{c}1 \\
0.1 \%\end{array}$ & $\begin{array}{l}\text { Correct } \rightarrow 94.7 \% \\
\text { Wrong } \rightarrow 5.3 \%\end{array}$ \\
\hline
\end{tabular}

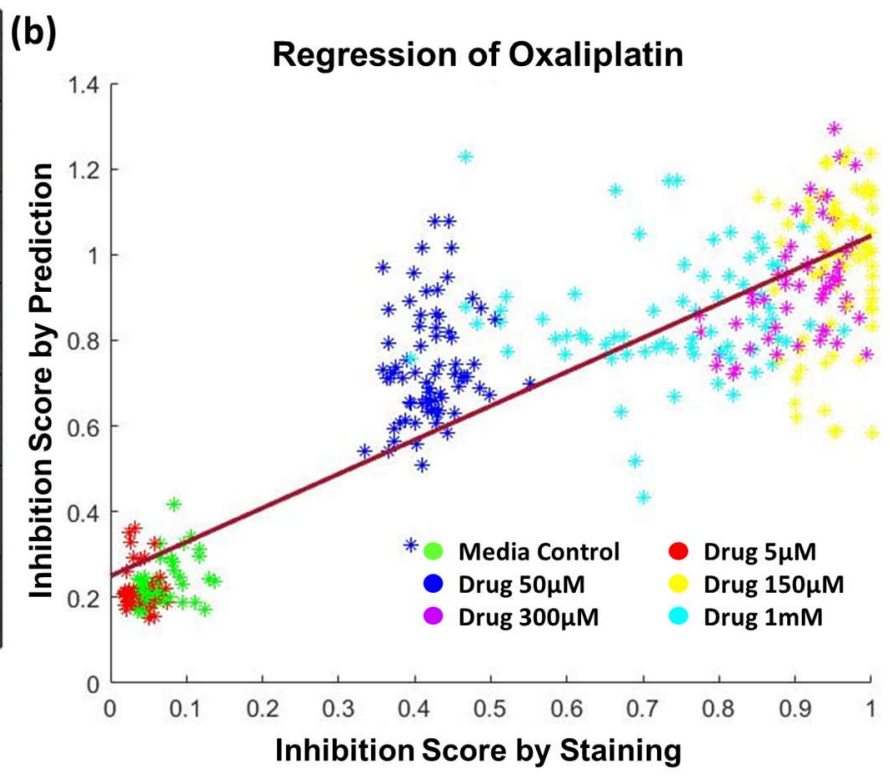

Figure S4. Cancer spheres classification and drug inhibition score regression for Oxaliplatin. (a) Sixclass classification accurately $(83.9 \%)$ predicts the drug treatment concentration based on its bright-field image. (b) Regression of sphere inhibition score using the trained CNN model. X-axis represents the ground truth of inhibition score measured by LIVE/DEAD staining, and Y-axis represents drug inhibition score predicted using bright-field image and the trained model. Each dot indicates a sphere, and different colors mean different drug concentrations. The $\mathrm{R}$-value of this linear regression is 0.89 , indicating a strong correlation between the ground truth and our prediction. 


\begin{tabular}{|c|c|c|c|c|c|c|c|}
\hline (a) & $\begin{array}{c}188 \\
12.2 \%\end{array}$ & $\begin{array}{c}34 \\
2.2 \%\end{array}$ & $\begin{array}{c}\mathbf{2 5} \\
1.6 \%\end{array}$ & $\begin{array}{c}0 \\
0.0 \%\end{array}$ & $\begin{array}{c}0 \\
0.0 \%\end{array}$ & $\begin{array}{c}0 \\
0.0 \%\end{array}$ & $\begin{array}{l}76.1 \% \\
23.9 \%\end{array}$ \\
\hline$\sum_{j}$ & $\begin{array}{c}44 \\
2.9 \%\end{array}$ & $\begin{array}{c}123 \\
8.0 \%\end{array}$ & $\begin{array}{c}48 \\
3.1 \%\end{array}$ & $\begin{array}{c}0 \\
0.0 \%\end{array}$ & $\begin{array}{c}0 \\
0.0 \%\end{array}$ & $\begin{array}{c}\mathbf{0} \\
0.0 \%\end{array}$ & $\begin{array}{l}57.2 \% \\
42.8 \%\end{array}$ \\
\hline$\varliminf_{\text {I }}$ & $\begin{array}{c}40 \\
2.6 \%\end{array}$ & $\begin{array}{c}58 \\
3.8 \%\end{array}$ & $\begin{array}{c}\mathbf{1 8 6} \\
12.1 \%\end{array}$ & $\begin{array}{c}\mathbf{5} \\
0.3 \%\end{array}$ & $\begin{array}{c}3 \\
0.2 \%\end{array}$ & $\begin{array}{c}\mathbf{0} \\
0.0 \%\end{array}$ & $\begin{array}{l}63.7 \% \\
36.3 \%\end{array}$ \\
\hline$\sum_{\substack{0 \\
0}}$ & $\begin{array}{c}0 \\
0.0 \%\end{array}$ & $\begin{array}{c}1 \\
0.1 \%\end{array}$ & $\begin{array}{c}9 \\
0.6 \%\end{array}$ & $\begin{array}{c}\mathbf{2 2 8} \\
14.8 \%\end{array}$ & $\begin{array}{c}16 \\
1.0 \%\end{array}$ & $\begin{array}{c}\mathbf{0} \\
0.0 \%\end{array}$ & $\begin{array}{l}89.8 \% \\
10.2 \%\end{array}$ \\
\hline 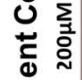 & $\begin{array}{c}0 \\
0.0 \%\end{array}$ & $\begin{array}{c}0 \\
0.0 \%\end{array}$ & $\begin{array}{c}0 \\
0.0 \%\end{array}$ & $\begin{array}{c}19 \\
1.2 \%\end{array}$ & $\begin{array}{c}\mathbf{2 3 0} \\
15.0 \%\end{array}$ & $\begin{array}{c}1 \\
0.1 \%\end{array}$ & $\begin{array}{l}92.0 \% \\
8.0 \%\end{array}$ \\
\hline 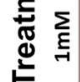 & $\begin{array}{c}0 \\
0.0 \%\end{array}$ & $\begin{array}{c}0 \\
0.0 \%\end{array}$ & $\begin{array}{c}0 \\
0.0 \%\end{array}$ & $\begin{array}{c}0 \\
0.0 \%\end{array}$ & $\begin{array}{c}7 \\
0.5 \%\end{array}$ & $\begin{array}{c}\mathbf{2 7 1} \\
17.6 \%\end{array}$ & $\begin{array}{c}97.5 \% \\
2.5 \%\end{array}$ \\
\hline $\begin{array}{l}\bar{\Xi} \\
\stackrel{\Xi}{\leftrightarrows}\end{array}$ & $\begin{array}{l}69.1 \% \\
30.9 \%\end{array}$ & $\begin{array}{l}56.9 \% \\
43.1 \%\end{array}$ & $\begin{array}{l}69.4 \% \\
30.6 \%\end{array}$ & $\begin{array}{c}90.5 \% \\
9.5 \%\end{array}$ & $\begin{array}{l}89.8 \% \\
10.2 \%\end{array}$ & $\begin{array}{c}99.6 \% \\
0.4 \%\end{array}$ & $\begin{array}{l}79.8 \% \\
20.2 \%\end{array}$ \\
\hline
\end{tabular}
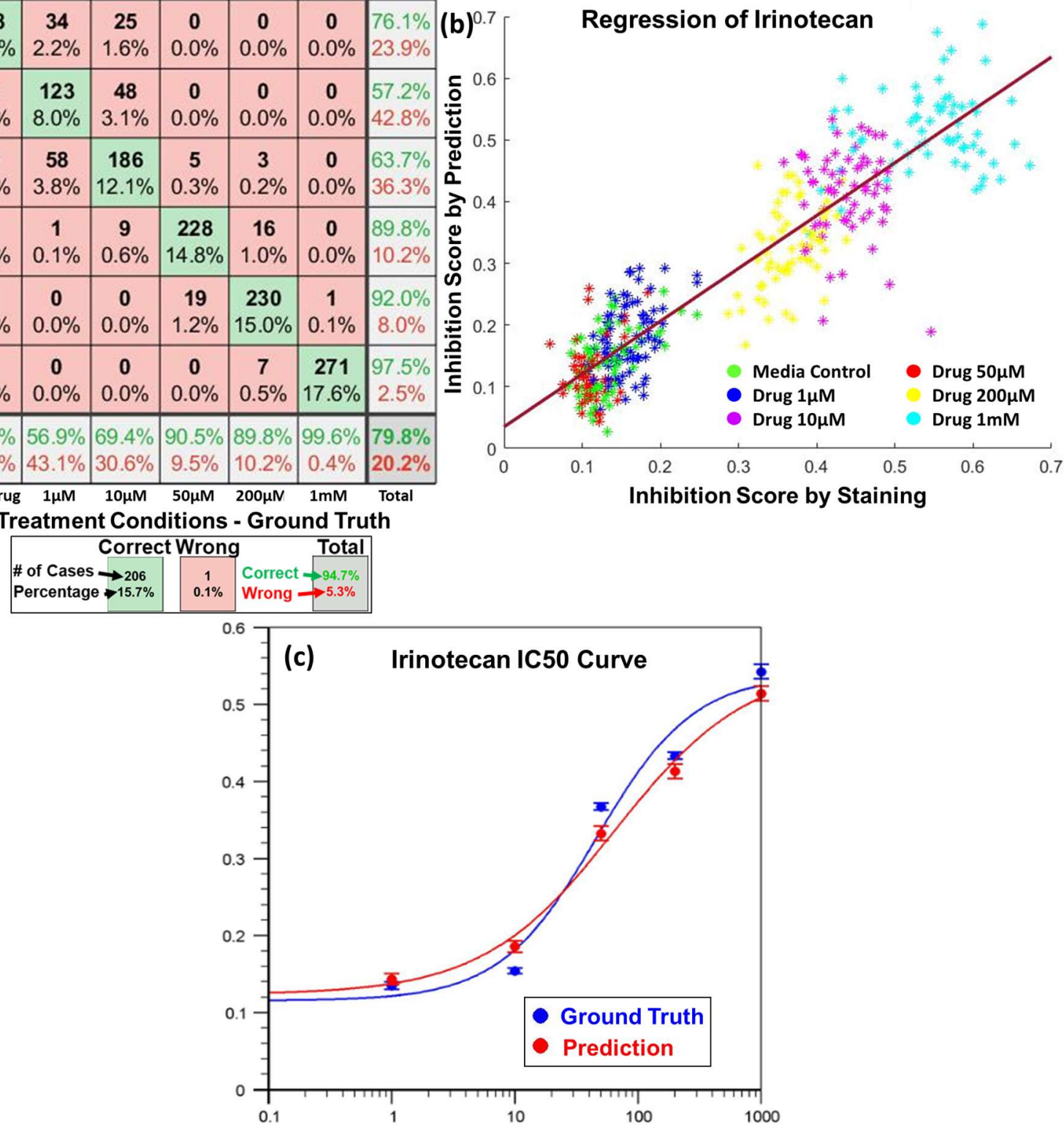

Figure S5. Cancer spheres classification and drug inhibition score regression for Irinotecan. (a) Sixclass classification accurately (79.8\%) predicts the drug treatment concentration based on its bright-field image. (b) Regression of sphere inhibition score using the trained CNN model. X-axis represents the ground truth of inhibition score measured by LIVE/DEAD staining, and Y-axis represents drug inhibition score predicted using bright-field image and the trained model. Each dot indicates a sphere, and different colors mean different drug concentrations. The $\mathrm{R}$-value of this linear regression is 0.91 , indicating a strong correlation between the ground truth and our prediction. (c) Drug efficacy IC50 curves of Irinotecan. Xaxis represents drug concentration, and Y-axis represents drug inhibition score. Blue curve is plotted based on the ground truth measured by LIVE/DEAD staining, and red curve is plotted based on the prediction using brightfield images. Error bars indicate standard error of the mean (SEM). Drug efficacy IC50 curves of Irinotecan treatment on cancer spheres. The estimated IC50 based on LIVE/DEAD staining is $46.6 \mu \mathrm{M}$, and the estimated IC50 based on brightfield images is $65.1 \mu \mathrm{M}$. 